\title{
Infection Control Nursing
}

National Cancer Institute

\section{Source}

National Cancer Institute. Infection Control Nursing. NCI Thesaurus. Code C19366.

A nursing specialty devoted to control and elimination of nosocomial infection risk in a

clinical setting. They do this through risk assessment, risk management, and staff education. 